\title{
Physical Activity and Fruit and Vegetable Intake among Black and White Female College Students at Two- and Four-Year Colleges and Universities
}

\author{
Carla J. Berg ${ }^{*}$, Sherell Brown Goodwin ${ }^{1}$, Erin Stratton ${ }^{1}$, Kincaid Lowe ${ }^{1}$, Linda Grimsley ${ }^{2}$, \\ Jan Rodd2, Catherine Williams'², Cheri Mattox ${ }^{3}$, Bruce Foster ${ }^{4}$ \\ ${ }^{1}$ Department of Behavioral Sciences and Health Education, Emory University, Atlanta, USA \\ ${ }^{2}$ Albany State University, Albany, USA \\ ${ }^{3}$ Chattahoochee Technical College, Marietta, USA \\ ${ }^{4}$ Central Georgia Technical College, Macon, USA \\ Email: ${ }^{*}$ cjberg@emory.edu
}

Received 3 February 2014; revised 19 March 2014; accepted 4 April 2014

Copyright (C) 2014 by authors and Scientific Research Publishing Inc.

This work is licensed under the Creative Commons Attribution International License (CC BY). http://creativecommons.org/licenses/by/4.0/

(c) (7) Open Access

\section{Abstract}

Objective: We compared fruit and vegetable (FV) intake, physical activity (PA), and overweight/ obesity among Black and White females attending two- and four-year colleges. Methods: We recruited 24,055 students at six colleges in the Southeast to complete an online survey, yielding 4840 responses $(20.1 \%$ response rate). The current analyses focused on the 2276 Black and White females. Results: Binary logistic regression analyses indicated that recommended FV intake among White females was associated with greater extraversion (Odds Ratio [OR] $=1.05,95 \%$ Confidence Interval $[\mathrm{CI}] 1.00,1.11, \mathrm{p}=\mathbf{0 . 0 5})$ and greater conscientiousness $(\mathrm{OR}=1.08, \mathrm{CI} 1.02,1.14, \mathrm{p}$ $=0.01$ ), whereas among Black females correlates included greater openness to experience $(\mathrm{OR}=$ 1.08, CI 1.01, 1.15, $p=0.03$ ). Ordinal logistic regression analyses indicated that, among White females, greater PA was associated with attending a four-year college (Beta $=0.27, \mathrm{CI} 0.01,0.53, \mathrm{p}=$ 0.04), whereas among Black females, correlates were with younger age $(B e t a=-0.01, C I-0.17$, $-0.03, p=0.003$ ) and greater emotional stability (Beta $=0.07$, CI $0.01,0.13, p=0.02$ ). Binary logistic regression analyses indicated that, among White females, being overweight or obese was associated with older age $(\mathrm{OR}=1.08, \mathrm{CI} 1.01,1.16, \mathrm{p}=0.03)$, attending a two-year college $(\mathrm{OR}=1.62, \mathrm{CI}$ $1.22,2.16, p=0.001)$, and lower satisfaction with life $(\mathrm{OR}=0.96, \mathrm{CI} 0.94,0.98, \mathrm{p}=0.002)$, whereas among Black females, being overweight or obese was associated with older age $(O R=1.87, \mathrm{CI} 1.10$, $1.28, p<0.001)$. Conclusions: Identifying factors related to obesity-related factors is critical in de-

\footnotetext{
${ }^{*}$ Corresponding author.
}

How to cite this paper: Berg, C.J., et al. (2014) Physical Activity and Fruit and Vegetable Intake among Black and White Female College Students at Two- and Four-Year Colleges and Universities. Open Journal of Preventive Medicine, 4, $229-239$. 
veloping interventions targeting factors associated with overweight and obesity among Black and White females attending two- and four-year colleges. Moreover, understanding different college settings and the contextual factors associated with overweight/obesity is critical.

\title{
Keywords
}

\author{
Obesity; Physical Activity; Nutrition; College Students; Female Health
}

\section{Introduction}

Chronic diseases such as cancer, heart disease and stroke account for more than $50 \%$ of all deaths among Americans each year [1]. By 2020, the rate of Americans affected by chronic disease is expected to grow to 157 million, with $51.5 \%$ of those affected having multiple conditions [2]. These conditions are often attributed to poor nutrition, lack of physical activity, tobacco use, and excessive alcohol consumption [1]. Modifying behaviors such as nutrition and physical activity has been shown to decrease the risk of cancer, heart disease, and diabetes [1]. Addressing these behaviors in adolescent and early adult years may have positive effects on health outcomes later in life.

Poor dietary and physical activity patterns commonly formed during the early adult years [3]-[5] may set the foundation for increased risk for chronic disease later in life [6], including osteoporosis, obesity, hyperlipedemia, and diabetes [7] [8]. College students' diets are typically low in fruits and vegetables [9]-[13] and high in fat [14] [15], sodium [14] [15], and added sugars [15]. This may be related to frequent eating out, time and monetary constraints, convenience, weight-control attitudes and habits, and misperceptions about nutrition [16]. Exercise and vigorous physical activity decline significantly from high school to college [17]. In fact, only $46 \%$ of college students report meeting the guidelines for physical activity which includes [9] at least 30 minutes of moderate intensity cardio or aerobic exercise on five or more days per week or at least 20 minutes of vigorous intensity cardio or aerobic exercise on three or more days per week [18]. Furthermore, $37 \%$ of the college student population is overweight, with $11 \%$ being obese [9]. College years are particularly important for behavioral change. During this time, students are impressionable, as they are in a new environment and primed for learning [19]. Thus, it is critical to encourage healthy eating early in life and through many different avenues in order to prevent the early onset of chronic illness.

Over 18 million students are enrolled in colleges and universities in the United States [20]. About 30\% of these students attend two-year colleges (i.e., community colleges) rather than traditional four-year universities, and this number has been increasing [20]. In comparison to students in four-year colleges, these students are more likely to be minority students, have fewer socioeconomic resources, and have work and family responsibilities [21]. In addition, given that two-year colleges tend to focus less on health in their curricula and have limited campus-based health services these students are less likely to receive important health information [22]. This disparity in health education and resources is concerning given the known relationships of lower educational level and lower socioeconomic status to physical activity, nutrition, chronic conditions, and shorter life expectancy [23]-[26]. Despite the fact that many young adults attend these two-year colleges and may have a higher health-risk profile [16], research has disproportionately focused on four-year universities. Thus, investigating differences in nutrition and physical activity and related psychosocial constructs among two-year and four-year colleges is critical.

In addition, females and males differ dramatically in their nutritional intake and level of physical activity [23]-[26]. In regard to diet, it is documented that males tend to be less concerned about dietary fat intake than females [27]. However, males do engage in exercise more frequently than females during the college years [28]. Furthermore, the prevalence of obesity in female adolescents and young adults differs by race, with Black females more commonly being obese and less frequently engaging in physical activity [29] [30].

Given the distinct differences in the genders and the importance of racial differences, the present study focuses on non-Hispanic Black and White females and investigates the differences in level of fruit and vegetable (FV) intake, physical activity (PA), and body mass index (BMI) among non-Hispanic White and Black females attending two- and four-year colleges and universities in the Southeastern US. We will also examine correlates of 
recommended FV intake, physical activity, and BMI among these female college students. Understanding these differences may provide essential information for designing effective interventions for improving nutrition and physical activity among potentially high-risk, underserved female young adults.

\section{Methods}

\subsection{Procedures}

In October, 2010, students at six colleges in the Southeast were recruited to complete an online survey (for more details, see [31]). A random sample of 5000 students at each school (with the exclusion of two schools who had enrollment less than 5000) were invited to complete the survey (total invited $N=24,055$ ). Students received an e-mail containing a link to the consent form with the alternative of opting out. The consent form included information about the study (i.e., cross-sectional survey about college student health), including the fact that their participation was strictly voluntary and that they could withdraw at any time without penalty. Students who consented to participate were directed to the online survey. To encourage participation, students received up to three e-mail invitations to participate. As an incentive for participation, all students who completed the survey received entry into a drawing for cash prizes of $\$ 1000$ (one prize), $\$ 500$ (two prizes), and $\$ 250$ (four prizes) at each participating school. Of students who received the invitation to participate, 4849 (20.1\%) returned a completed survey. The current analyses focused on the 2276 participants who reported being female and of nonHispanic White or Black ethnicity. The Emory University Institutional Review Board approved this study, IRB\# 00030631.

\subsection{Measures}

Sociodemographic Characteristics. We assessed students' age, gender, ethnicity, and type of school attended (two-year vs four-year). Ethnicity was categorized as non-Hispanic White, Black, or Other due to the small numbers of participants who reported other race/ethnicities, and the current analyses focused on only those participants who reported being non-Hispanic White or Black and reported being female.

Nutrition. To assess dietary nutrition, we asked three questions: 1) "Over the past 7 days, on average how many servings of fruit did you eat per day?”; 2) “Over the past 7 days, on average how many servings of vegetables did you eat per day?”; and 3) "Overall, when you think about the foods you ate over the past 12 months, would you say that your diet was low, medium, or high in fat? Low, Medium, or High.” Based on the recommendations for appropriate fruit and vegetable (FV) intake [26], we dichotomized the FV variable as $<5$ servings of FV per week vs $\geq 5$ servings of FV per week. We examined self-reported level of dietary fat intake as a correlate of the other outcomes of interest.

Physical Activity. To assess physical activity (PA), participants were asked : 1) "During the past 7 days, on how many of those days did you do moderate-intensity cardio or aerobic exercise (caused a noticeable increase in heart rate, such as a brisk walk) for at least 30 minutes?”; 2) "During the past 7 days, on how many of those days did you do vigorous-intensity cardio or aerobic exercise (caused large increases in breathing or heart rate, such as jogging) for at least 20 minutes?”; and 3) "During the past 7 days, on how many of those days did you do 8 - 10 strength training exercises (such as resistance weight machines) for 8 - 12 repetitions?” We classified participants not indicating any of the assessed activities in the past 7 days as sedentary, those who engaged in less than five days of cardio or aerobic exercise and less than two days of strength training as having insufficient physical activity, and those who engaged in at least five days of cardio or aerobic exercise and at least two days of strength training as having sufficient physical activity, based on CDC recommendations [32].

Weight and Weight Concern. We also assessed height and weight and calculated body mass index (BMI) and categorized individuals into underweight $(<18.5)$, normal weight (18.5 to 24.9), overweight (25.0 to 29.9), and obese $(\geq 30)$. We then collapsed this variable in underweight/normal weight vs overweight/obese for ease of interpretation of results. We also asked, "How do you describe your weight?" with response options of Very underweight, Slightly underweight, About the right weight, Slightly overweight, or Very overweight. To examine weight-related concern, we asked, "Are you trying to do any of the following about your weight?" with response options of "I am not trying to do anything about my weight, Stay the same weight, Lose weight, or Gain weight."

Psychosocial Factors. To assessed perceived stress, we administered the Perceived Stress Scale (PSS-4) [33] to assess the amount of stress they experienced in the past month. Higher total scores indicate greater levels of 
perceived stress. To assess satisfaction with life, we administered the Satisfaction with Life Scale [34], a fiveitem scale containing items such as "In most ways my life is close to my ideal" and "If I could live my life over, I would change almost nothing" on a scale of $1=$ strongly disagree to $7=$ strongly agree. The scale demonstrates appropriate validity and reliability (Cronbach's alpha of 0.87 ). We also administered the Ten-Item Personality Inventory (TIPI) [35], which organizes personality theories into five primary non-cognitive personality factors, called the "Big Five" (Extraversion, Agreeableness, Conscientiousness, Emotional Stability, and Openness to Experience). Items are rated on a 7-point scale ( 1 = disagree strongly to 7 = agree strongly). The TIPI has demonstrated appropriate internal consistency, adequate convergent validity, test-retest reliability, and appropriate patterns of predicted external correlates [35].

\subsection{Data Analysis}

Participant characteristics were summarized using means and standard deviations and $\mathrm{N}$ and percentages. Chisquared analyses were used to examine differences in obtaining the recommended FV intake, PA, and BMI between White and Black female college students. In addition, we conducted bivariate analyses to identify correlates of obtaining the recommended FV intake, PA, and BMI among White and Black females separately. Finally, six logistic regression models were conducted examining factors related to level of FV intake, PA, and BMI among White and Black female college students separately. We forced age, type of school, and the psychosocial factors into each equation to identify sociodemographic and psychosocial factors associated with recommended FV intake, PA, and BMI among the female participants. Significance was set at p $=0.05$, and all analyses were conducted using SPSS version 21.0.

\section{Results}

Our sample included 48.7\% $(n=1109)$ White and 51.3\% $(n=1167)$ Black females. Across all three outcomes of interest (FV intake, PA, and BMI), White females were more likely than Black females to consume the recommended level of FV $(p=0.01)$, to engage in the recommended PA $(\mathrm{p}<0.001)$, and report being under weight or of normal weight $(\mathrm{p}<0.001$; see Tables $1-3)$.

\subsection{Fruit and Vegetable Intake}

Table 1 presents the correlates of consuming 5 or more FV per day in the past 7 days. Among White females, bivariate analyses indicated that consuming $\geq 5 \mathrm{FV}$ per day in the past 7 days was associated with greater extraversion ( $p=0.02)$, greater conscientiousness $(p=0.004)$, limiting dietary fat intake $(p<0.001)$, and higher level of PA ( $<$ 0.001). Among Black females, bivariate analyses indicated that requisite FV intake was associated with self-reported weight $(p=0.005)$, actions toward weight management $(p=0.04)$, limiting dietary fat intake $(\mathrm{p}<0.001)$, higher level of PA $(\mathrm{p}<0.001)$, and higher BMI $(\mathrm{p}<0.001)$.

Binary logistic regression analyses indicated that recommended FV intake among White females was associated with greater extraversion (Odds Ratio $[\mathrm{OR}]=1.05$, 95\% Confidence Interval $[\mathrm{CI}] 1.00,1.11, \mathrm{p}=0.05$ ) and greater conscientiousness $(\mathrm{OR}=1.08, \mathrm{CI} 1.02,1.14, \mathrm{p}=0.01$; Nagelkerke R-squared $=0.019)$. Multivariate analyses indicated that recommended FV intake among Black females included greater openness to experience $(\mathrm{OR}=1.08, \mathrm{CI} 1.01,1.15, \mathrm{p}=0.03$; Nagelkere R-squared $=0.016)$.

\subsection{Physical Activity}

Table 2 displays bivariate analyses of White and Black females related to level of PA. Bivariate analyses indicated that level of PA among White females included attending a four-year school $(\mathrm{p}=0.01)$, lower perceived stress $(p=0.04)$, greater satisfaction with life $(p=0.04)$, greater extraversion $(p=0.05)$, greater emotional stability ( $\mathrm{p}=0.002)$, self-reported weight $(\mathrm{p}=0.008)$, actions toward weight management $(\mathrm{p}<0.001)$, lower dietary fat intake $(\mathrm{p}<0.001)$, greater FV consumption $(\mathrm{p}<0.001)$, and lower BMI $(\mathrm{p}=0.10)$. Among Black females, bivariate analyses indicated that PA level was associated with being younger $(p=0.006)$, greater satisfaction with life $(p=0.03)$, greater emotional stability $(p=0.05)$, actions toward weight management $(p=0.03)$, limiting dietary fat $(\mathrm{p}<0.001)$, and lower BMI $(\mathrm{p}<0.001)$.

Ordinal logistic regression analyses indicated that, among White females, greater PA was associated with being attending a four-year college (Beta $=0.27$, CI 0.01, 0.53, $\mathrm{p}=0.04$; Nagelkerke R-squared $=0.029$ ). Among 
Table 1. Participant characteristics and bivariate analyses examining factors associated with average daily fruit and vegetable intake in the past week among college females.

\begin{tabular}{|c|c|c|c|c|c|c|c|}
\hline \multirow[b]{2}{*}{ Variable } & \multirow{2}{*}{$\begin{array}{l}\text { Total } \\
\text { N (\%) or } \\
\text { M (SD) }\end{array}$} & \multicolumn{2}{|c|}{ White $(n=1049)$} & \multirow[b]{2}{*}{$\mathrm{p}$} & \multicolumn{2}{|c|}{ Black $(n=1083)$} & \multirow[b]{2}{*}{$\mathrm{p}$} \\
\hline & & $\begin{array}{c}<5 \\
\mathrm{~N}(\%) \text { or } \\
\mathrm{M}(\mathrm{SD})\end{array}$ & $\begin{array}{c}\geq 5 \\
\mathrm{~N}(\%) \text { or } \\
\mathrm{M} \text { (SD) }\end{array}$ & & $\begin{array}{c}<5 \\
\mathrm{~N}(\%) \text { or } \\
\mathrm{M}(\mathrm{SD})\end{array}$ & $\begin{array}{c}\geq 5 \\
\mathrm{~N}(\%) \text { or } \\
\mathrm{M}(\mathrm{SD})\end{array}$ & \\
\hline Total & $2132(100.0)$ & $386(36.8)$ & $663(63.2)$ & * & $450(41.6)$ & $633(58.4)$ & * \\
\hline \multicolumn{8}{|l|}{ Sociodemographics } \\
\hline Age (SD) & $20.70(2.02)$ & $20.68(1.96)$ & $663(20.72)$ & 0.76 & $20.24(1.85)$ & $20.33(1.88)$ & 0.44 \\
\hline College type (\%) & & & & 0.92 & & & 0.20 \\
\hline Four-year colleges & $619(59.0)$ & $227(58.8)$ & $392(59.1)$ & & $387(86.0)$ & $526(83.1)$ & \\
\hline Two-year colleges & $430(41.0)$ & $159(41.2)$ & 271 (40.9) & & $63(14.0)$ & 107 (16.9) & \\
\hline \multicolumn{8}{|l|}{ Psychosocial variables } \\
\hline Perceived stress (SD) & $6.35(3.40)$ & $6.52(3.41)$ & $6.25(3.40)$ & 0.29 & $6.35(3.38)$ & $6.26(3.33)$ & 0.66 \\
\hline Satisfaction with life (SD) & $23.25(7.42)$ & $23.00(4.43)$ & $23.40(7.42)$ & 0.42 & $22.46(7.37)$ & $22.77(7.25)$ & 0.53 \\
\hline \multicolumn{8}{|l|}{ Big 5 personality traits (SD) } \\
\hline Extraversion & $9.18(2.93)$ & $8.88(2.95)$ & $9.35(2.91)$ & 0.02 & $8.73(2.86)$ & $8.71(2.72)$ & 0.92 \\
\hline Agreeableness & $10.11(2.25)$ & $10.10(2.33)$ & $10.12(2.21)$ & 0.92 & $10.19(2.31)$ & $10.01(2.29)$ & 0.26 \\
\hline Conscientiousness & $11.12(2.45)$ & $10.82(2.55)$ & $11.29(2.36)$ & 0.004 & $11.55(2.21)$ & $11.32(2.41)$ & 0.14 \\
\hline Emotional stability & $8.84(2.74)$ & $8.64(2.78)$ & $8.95(2.72)$ & 0.09 & $9.87(2.62)$ & $9.65(2.73)$ & 0.21 \\
\hline Openness & $10.77(2.23)$ & $10.68(2.26)$ & $10.83(2.21)$ & 0.32 & $10.94(2.31)$ & $11.45(2.21)$ & 0.18 \\
\hline \multicolumn{8}{|l|}{ Weight-related variables } \\
\hline Weight self-report (\%) & & & & 0.24 & & & 0.005 \\
\hline Underweight & $67(6.4)$ & $29(7.5)$ & $38(5.7)$ & & $45(10.0)$ & $41(6.5)$ & \\
\hline About right & $535(51.0)$ & $185(47.9)$ & $350(52.8)$ & & $214(47.6)$ & $266(42.0)$ & \\
\hline Overweight & $447(42.6)$ & $172(44.6)$ & $275(41.5)$ & & $191(42.4)$ & $326(51.5)$ & \\
\hline Actions toward weight management (\%) & & & & 0.64 & & & 0.04 \\
\hline Lose weight & $615(58.6)$ & $224(58.0)$ & $391(59.0)$ & & $240(53.3)$ & $387(61.1)$ & \\
\hline Maintain weight/no action & $410(39.1)$ & $151(39.1)$ & $259(39.1)$ & & $161(35.8)$ & 189 (29.9) & \\
\hline Gain weight & $24(2.3)$ & $11(2.8)$ & $13(2.0)$ & & 49 (10.9) & $57(9.0)$ & \\
\hline Dietary fat intake (\%) & & & & $<0.001$ & & & $<0.001$ \\
\hline Low & $123(11.7)$ & $27(7.0)$ & $96(14.5)$ & & $36(8.0)$ & $52(8.2)$ & \\
\hline Medium & 729 (69.5) & 265 (68.7) & $464(70.0)$ & & $244(54.2)$ & $421(66.5)$ & \\
\hline High & 195 (18.8) & $94(24.4)$ & $103(15.5)$ & & $170(37.8)$ & $160(25.3)$ & \\
\hline Physical activity (\%) & & & & $<0.001$ & & & $<0.001$ \\
\hline Sedentary & 265 (25.5) & $109(28.8)$ & $156(23.6)$ & & $197(44.9)$ & $187(29.7)$ & \\
\hline Insufficient & 369 (55.8) & $235(62.0)$ & 369 (55.8) & & $202(46.0)$ & $346(55.0)$ & \\
\hline Sufficient & $136(20.6)$ & $35(9.2)$ & $136(20.6)$ & & $40(9.1)$ & 96 (15.3) & \\
\hline BMI category (\%) & & & & 0.45 & & & $<0.001$ \\
\hline Normal or underweight & $700(66.9)$ & $263(68.3)$ & $437(66.0)$ & & $250(55.6)$ & $281(44.4)$ & \\
\hline Overweight or obese & 347 (33.1) & 122 (31.7) & $225(34.0)$ & & $200(44.4)$ & $352(55.6)$ & \\
\hline
\end{tabular}

" ${ }^{*}$-value for Chi-squared analyses among all black and white females in reference to FV intake $=0.01$. 
Table 2. Participant characteristics and bivariate analyses examining factors associated with physical activity level among college females.

\begin{tabular}{|c|c|c|c|c|c|c|c|c|c|}
\hline \multirow[b]{2}{*}{ Variable } & \multirow[b]{2}{*}{$\begin{array}{c}\text { Total } \\
\mathrm{N}(\%) \text { or } \\
\mathrm{M}(\mathrm{SD})\end{array}$} & \multicolumn{3}{|c|}{ White $(\mathrm{n}=1040)$} & \multirow[b]{2}{*}{$\mathrm{p}$} & \multicolumn{3}{|c|}{ Black $(\mathrm{n}=1071)$} & \multirow[b]{2}{*}{$\mathrm{p}$} \\
\hline & & $\begin{array}{l}\text { Sedentary } \\
\text { N (\%) or } \\
\text { M (SD) }\end{array}$ & $\begin{array}{l}\text { Insufficient } \\
\mathrm{N}(\%) \text { or } \\
\text { M (SD) }\end{array}$ & $\begin{array}{l}\text { Sufficient } \\
\text { N (\%) or } \\
\text { M (SD) }\end{array}$ & & $\begin{array}{l}\text { Sedentary } \\
\mathrm{N}(\%) \text { or } \\
\mathrm{M}(\mathrm{SD})\end{array}$ & $\begin{array}{l}\text { Insufficient } \\
\text { N (\%) or } \\
\text { M (SD) }\end{array}$ & $\begin{array}{l}\text { Sufficient } \\
\text { N (\%) or } \\
\text { M (SD) }\end{array}$ & \\
\hline Total & $2111(100.0)$ & $265(25.5)$ & 604 (58.1) & $171(16.4)$ & ${ }^{*}$ & 385 (35.9) & 549 (51.3) & 137 (12.8) & \\
\hline
\end{tabular}

Sociodemographics

Age (SD)

College type (\%)

Four-year colleges

Two-year colleges

Psychosocial variables

Perceived stress (SD)

Satisfaction with life (SD)

Big 5 personality traits (SD)

Extraversion

Agreeableness

Conscientiousness

Emotional stability

Openness

Weight-related variables

Weight self-report (\%)

Underweight

About right

Overweight

Actions toward weight management (\%)

Lose weight
Maintain weight/no action

Gain weight

Dietary fat intake (\%)

Low

Medium

High

FV consumption in past week (\%)

$$
0 \text { to }<5 \text { per day }
$$

5 or more per day

BMI category (\%)

Normal or underweight

Overweight or obese

20.70 (2.02) $20.80(2.07) 20.70(2.01) 20.50$ (1.97) $0.30 \quad 20.52(1.92) 20.23(1.84) \quad 19.97(1.81) \quad 0.006$ 0.01

0.22

$615(59.1) \quad 137(51.7) \quad 368(60.9) \quad 110(64.3) \quad 315(81.8) \quad 469(85.4) \quad 119(86.9)$

$425(40.9) \quad 128(48.3) \quad 236(39.1) \quad 61(35.7) \quad 70(18.2) \quad 80(14.6) \quad 18(13.1)$

$6.34(3.41) \quad 6.68(3.49) \quad 6.34(3.40) \quad 5.81(3.23) \quad 0.04 \quad 6.50(3.43) \quad 6.14(3.20) \quad 6.12(3.49) \quad 0.28$

23.28 (7.42) $22.26(7.66) \quad 23.55(7.40) \quad 23.87(7.02) \quad 0.04 \quad 21.79(7.63) 23.15(6.97) 23.00$ (7.57) $\quad 0.03$

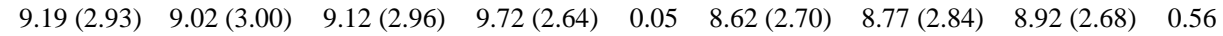

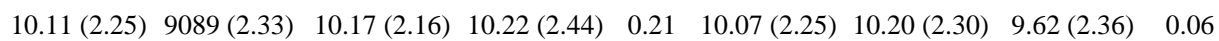

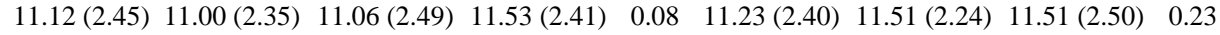
$8.85(2.73) \quad 8.45(2.84) \quad 8.87(2.73) \quad 9.43(2.43) \quad 0.002 \quad 9.53(2.73) \quad 9.77(2.57) \quad 10.24(2.77) \quad 0.05$ 10.77 (2.23) $10.65(2.23) 10.74(2.16) 11.10(2.44) \quad 0.13 \quad 10.97(2.24) 11.12(2.26) 11.14(2.24) \quad 0.59$

\section{(1)}


Table 3. Participant characteristics and bivariate analyses examining factors associated with body mass index (BMI) among college females.

\begin{tabular}{|c|c|c|c|c|c|c|c|}
\hline \multirow[b]{2}{*}{ Variable } & \multirow[b]{2}{*}{$\begin{array}{c}\text { Total } \\
\text { N (\%) or } \\
\text { M (SD) }\end{array}$} & \multicolumn{2}{|c|}{ White $(\mathrm{n}=1109)$} & \multirow[b]{2}{*}{$\mathrm{p}$} & \multicolumn{2}{|c|}{ Black $(n=1167)$} & \multirow[b]{2}{*}{$\mathrm{p}$} \\
\hline & & $\begin{array}{l}\text { Normal or } \\
\text { underweight } \\
\mathrm{N}(\%) \text { or } \\
\text { M (SD) }\end{array}$ & $\begin{array}{l}\text { Overweight } \\
\text { or obese } \\
\mathrm{N}(\%) \text { or } \\
\text { M (SD) }\end{array}$ & & $\begin{array}{l}\text { Normal or } \\
\text { underweight } \\
\text { N (\%) or } \\
\text { M (SD }\end{array}$ & $\begin{array}{l}\text { Overweight } \\
\text { or obese } \\
\text { N (\%) or } \\
\text { M (SD) }\end{array}$ & \\
\hline Total & $2276(100.0)$ & $748(67.4)$ & $361(32.5)$ & * & $571(48.9)$ & $596(51.1)$ & * \\
\hline \multicolumn{8}{|l|}{ Sociodemographics } \\
\hline Age (SD) & $20.69(2.03)$ & $20.55(1.97)$ & $20.99(2.11)$ & 0.001 & $20.02(1.82)$ & $20.54(1.90)$ & $<0.001$ \\
\hline College type (\%) & & & & $<0.001$ & & & 0.20 \\
\hline Four-year colleges & $645(58.2)$ & 472 (63.1) & $173(47.9)$ & & $488(85.5)$ & 493 (82.7) & \\
\hline Two-year colleges & $464(41.8)$ & $276(36.9)$ & $188(52.1)$ & & $83(14.5)$ & $103(17.3)$ & \\
\hline \multicolumn{8}{|l|}{ Psychosocial variables } \\
\hline Perceived stress (SD) & $6.35(3.41)$ & $6.16(3.36)$ & $6.73(3.48)$ & 0.01 & $6.21(3.42)$ & $6.37(3.30)$ & 0.47 \\
\hline Satisfaction with life (SD) & $23.25(7.43)$ & $23.98(7.07)$ & $21.76(7.91)$ & $<0.001$ & $23.02(7.54)$ & $22.26(7.05)$ & 0.11 \\
\hline \multicolumn{8}{|l|}{ Big 5 personality traits (SD) } \\
\hline Extraversion & 9.19 (2.93) & $9.13(2.89)$ & $9.31(3.00)$ & 0.38 & $8.55(2.80)$ & $8.89(2.76)$ & 0.06 \\
\hline Agreeableness & $1011(2.25)$ & $10.09(2.25)$ & $10.15(2.26)$ & 0.72 & $10.12(2.33)$ & $10.05(2.27)$ & 0.61 \\
\hline Conscientiousness & $11.12(2.44)$ & $11.21(2.42)$ & $10.92(2.48)$ & 0.09 & $11.44(2.28)$ & $11.40(2.38)$ & 0.79 \\
\hline Emotional stability & $8.84(2.74)$ & $8.92(2.62)$ & $8.92(2.62)$ & 0.17 & $9.64(2.67)$ & $9.84(2.71)$ & 0.26 \\
\hline Openness & $10.78(2.22)$ & $10.74(2.22)$ & $10.86(2.23)$ & 0.45 & $10.93(2.26)$ & $11.21(2.24)$ & 0.06 \\
\hline \multicolumn{8}{|l|}{ Weight-related variables } \\
\hline Weight self-report (\%) & & & & $<0.001$ & & & $<0.001$ \\
\hline Underweight & $67(6.4)$ & $64(9.1)$ & $3(0.9)$ & & $75(14.1)$ & $11(2.0)$ & \\
\hline About right & $535(51.1)$ & $505(72.1)$ & $30(8.6)$ & & $402(75.4)$ & $80(14.5)$ & \\
\hline Overweight & $445(42.5)$ & $131(18.7)$ & $314(90.5)$ & & $56(10.5)$ & $462(83.5)$ & \\
\hline Actions toward weight management (\%) & & & & $<0.001$ & & & $<0.001$ \\
\hline Lose weight & $613(58.5)$ & $331(47.3)$ & $282(81.3)$ & & $153(28.7)$ & $475(85.9)$ & \\
\hline Maintain weight/no action & $410(39.2)$ & $345(49.3)$ & $65(18.7)$ & & $277(52.0)$ & 75 (13.6) & \\
\hline Gain weight & $24(2.3)$ & $24(3.4)$ & $0(0.0)$ & & 103 (19.3) & $3(0.5)$ & \\
\hline Dietary fat intake (\%) & & & & $<0.001$ & & & $<0.001$ \\
\hline Low & 123 (11.7) & 95 (13.6) & $28(8.1)$ & & 52 (9.8) & $36(6.5)$ & \\
\hline Medium & $727(69.4)$ & 499 (71.3) & $228(65.7)$ & & 351 (65.9) & $316(57.1)$ & \\
\hline High & $197(18.8)$ & $106(15.1)$ & $91(26.2)$ & & $130(24.4)$ & $201(36.3)$ & \\
\hline FV consumption in past week (\%) & & & & 0.45 & & & $<0.001$ \\
\hline 0 to $<5$ per day & 385 (36.8) & $263(37.6)$ & 122 (35.2) & & $250(47.1)$ & $200(36.2)$ & \\
\hline 5 or more per day & 662 (63.2) & 437 (62.4) & 225 (64.8) & & 281 (52.9) & 352 (63.8) & \\
\hline Physical activity (\%) & & & & 0.02 & & & 0.10 \\
\hline Sedentary & 265 (25.5) & $167(24.0)$ & $98(28.6)$ & & 198 (37.7) & $187(34.2)$ & \\
\hline Insufficient & $602(58.0)$ & 398 (57.3) & 204 (59.5) & & 271 (51.6) & 278 (50.9) & \\
\hline Sufficient & $171(16.5)$ & 130 (18.7) & $41(12.0)$ & & 56 (10.7) & 81 (14.8) & \\
\hline
\end{tabular}

${ }^{*}$ p-value for Chi-squared analyses among all black and white females in reference to BMI $<0.001$. 
Black females, greater PA was associated with younger age (Beta $=-0.01, \mathrm{CI}-0.17,-0.03, \mathrm{p}=0.003$ ) and greater emotional stability (Beta $=0.07$, CI $0.01,0.13, \mathrm{p}=0.02$, Nagelkerke R-squared $=0.029$ ).

\subsection{Body Mass Index}

Table 3 displays bivariate analyses related to BMI level. Bivariate analyses indicated that, among White females, being overweight or obese was associated with older age $(p=0.001)$, attending a two-year college $(p<0.001)$, greater perceived stress $(\mathrm{p}=0.01)$, lower satisfaction with life $(\mathrm{p}<0.001)$, self-reported weight status $(\mathrm{p}<$ 0.001 ), actions toward weight management ( $<<0.001)$, higher level of dietary fat intake ( $<<0.001)$, and level of PA ( $\mathrm{p}=0.02)$. Among Black females, being overweight or obese was associated with being older $(\mathrm{p}<0.001)$, self-reported weight $(\mathrm{p}<0.001)$, actions toward weight change $(\mathrm{p}<0.001)$, higher dietary fat content $(\mathrm{p}<$ $0.001)$, and greater FV intake ( $<<0.001)$.

Binary logistic regression analyses indicated that, among White females, being overweight or obese was associated with older age $(\mathrm{OR}=1.08, \mathrm{CI} 1.01,1.16, \mathrm{p}=0.03)$, attending a two-year college $(\mathrm{OR}=1.62$, CI 1.22 , 2.16, $\mathrm{p}=0.001)$, and lower satisfaction with life $(\mathrm{OR}=0.96$, CI 0.94, 0.98, $\mathrm{p}=0.002$; Nagelkerke R-squared $=$ 0.062). Among Black females, being overweight or obese was associated with older age (OR $=1.87$, CI 1.10, 1.28, $\mathrm{p}<0.001$; Nagelkerke R-squared $=0.059$ ).

\section{Discussion}

This study documented differences in health behaviors among White and Black female college students in the Southeastern US Statistics regarding FV intake, PA level, and BMI indicated that White females in this sample demonstrated higher prevalence of engaging in the recommended FV intake and PA and lower prevalence of overweight and obesity. This study also documented unique correlates of appropriate health behaviors among these groups of college females.

Among White females, important correlates of achieving the recommended FV intake included higher levels of extraversion and conscientiousness. Perhaps more conscientious students are more likely to attend to their nutrition, and it is possible that more extraverted individuals also engage in more social interactions that provide opportunities for consuming diets richer in fruits and vegetables. The only factor associated with engaging in the recommended PA included attending a four-year college, which may be due to the more numerous opportunities to be involved in group or school-sponsored sports or the greater efforts to educate four-year college students on the importance of physical activity [22]. Correlates of being overweight or obese included being older, attending a two-year college, and lower satisfaction with life. Again, being older and attending two-year colleges are factors associated with obesity-related factors [17] [22]. Interestingly, satisfaction with life was uniquely associated with being overweight or obese in White females, indicating that there may be important cultural differences related to weight and race among females [36].

Among Black females, important correlates of achieving the recommended FV intake included higher levels of openness to experience. This interestingly has been found in a sample of Dutch adolescents [37]. The only factors associated with engaging in the recommended PA included being younger, which has been previously documented [17] and greater emotional stability. In reference to the latter finding, perhaps emotional stability leads to engagement in sufficient levels of PA or vice versa. Longitudinal studies are needed to clarify this association. Correlates of being overweight or obese included being older, which has been previously documented [17].

These specific associations between sociodemographic and psychosocial factors and FV intake, PA, and weight status among Black and White females attending two- and four-year colleges may have significant implications for research and practice. First, differences in health behaviors among these groups highlight the importance of including two-year college students in population-based assessments of health risk. Moreover, these differences might be a reflection of the variability that likely exists among young adults in the broader context. Thus, future research is encouraged to target other segments of the young adult population, such as those not enrolling in college (e.g., in the workforce or military). Second, understanding the health behaviors among these different groups as well as other psychosocial variables (e.g. attitudes, motives) that contribute to different behavioral patterns is critical for developing effective interventions to increase positive health-related behaviors. Finally, it is important to understand the context in which healthy behaviors occur (or do not occur), as the life circumstances for community college students differ compared to other young adults. For example, these com- 
munity college students have limited access to health education and campus-based health care. Overall, this study highlights the need for future research investigating these differences and interventions designed to address nutrition and physical activity among different segments of the college student population.

\subsection{Limitations}

Limitations to this study include limited generalizability due to recruitment at six colleges in the Southeast. An additional limitation is the low response rate (20.1\%), which may suggest response bias. However, previous research has found that the average email survey response rate is $24 \%$, which is only slightly higher than the response rate for this survey [38]. In addition, it is possible that some recruited students did not open the e-mail or had inactive accounts, which would influence the response rate. However, this cannot be assessed in the current study. Furthermore, previous research has indicated that, despite lower response rates, internet surveys yield similar data regarding health behaviors compared to mail and phone surveys [39]. Despite these limitations, this study provides strong support for continued research on different trajectories of smoking among college students.

\subsection{Conclusion}

Recommended FV intake among White females was associated with greater extraversion and greater conscientiousness, whereas among Black females correlates included greater openness to experience. Moreover, among White females, greater PA was associated with attending a four-year college, whereas among Black females, correlates were with younger age and greater emotional stability. Among White females, being overweight or obese was associated with older age, attending a two-year college, and lower satisfaction with life, whereas among Black females, being overweight or obese was associated with older age. Identifying factors related to obesity-related factors is critical in developing interventions targeting factors associated with overweight and obesity among Black and White females attending two- and four-year colleges. Moreover, understanding different college settings and the contextual factors associated with overweight/obesity is critical.

\section{Acknowledgements}

We would like to thank our collaborators across the state of Georgia in developing and administering this survey.

\section{Competing Interests}

The authors declare no conflicts of interest.

\section{Funding}

This research was supported by the National Cancer Institute (1K07CA139114-01A1; PI: Berg) and the Georgia Cancer Coalition (PI: Berg).

\section{References}

[1] Centers for Disease, C. and Prevention (2010) Vital Signs: State-Specific Obesity Prevalence among Adults—United States, 2009. MMWR Morbidity and Mortality Weekly Report, 59, 951-955.

[2] (2009) Tackling the Burden of Chronic Diseases in the USA. Lancet, 373, 185. http://dx.doi.org/10.1016/S0140-6736(09)60048-9

[3] Kolodinsky, J., et al. (2007) Knowledge of Current Dietary Guidelines and Food Choice by College Students: Better Eaters Have Higher Knowledge of Dietary Guidance. Journal of the American Dietetic Association, 107, 1409-1413. http://dx.doi.org/10.1016/j.jada.2007.05.016

[4] Larson, N.I., et al. (2007) Trends in Adolescent Fruit and Vegetable Consumption, 1999-2004: Project EAT. American Journal of Preventive Medicine, 32, 147-150. http://dx.doi.org/10.1016/j.amepre.2006.10.011

[5] Von Ah, D., et al. (2004) Predictors of Health Behaviours in College Students. Journal of Advanced Nursing, 48, 463474. http://dx.doi.org/10.1111/j.1365-2648.2004.03229.x

[6] American Cancer Society (2002) Recommendations on Nutrition and Physical Activity. California Working Families 
Policy Summit, Oakland.

[7] Bazzano, L.A. (2006) The High Cost of Not Consuming Fruits and Vegetables. Journal of the American Dietetic Association, 106, 1364-1368. http://dx.doi.org/10.1016/j.jada.2006.06.021

[8] Hallal, P.C., et al. (2006) Adolescent Physical Activity and Health: A Systematic Review. Sports Medicine, 36, 10191030. http://dx.doi.org/10.2165/00007256-200636120-00003

[9] The American College Health Association (2009) American College Health Association: National College Health Assessment Spring 2008 Reference Group Data Report (Abridged). Journal of American College Health, 57, 477-488. http://dx.doi.org/10.3200/JACH.57.5.477-488

[10] Melby, L., Femea, P. and Sciacca, J. (1986) Reported Dietary and Exercise Behaviors, Beliefs and Knowledge among University Undergraduates. Nutrition Research, 6, 799-808. http://dx.doi.org/10.1016/S0271-5317(86)80162-2

[11] Dinger, M.K. and Waigandt, A. (1997) Dietary Intake and Physical Activity Behaviors of Male and Female College Students. American Journal of Health Promotion, 11, 360-362. http://dx.doi.org/10.4278/0890-1171-11.5.360

[12] Hiza, H. and Gerrior, S. (2002) Using the Interactive Healthy Eating Index to Assess the Quality of College Students' Diets. Family Economics and Nutrition Review, 14, 3-11.

[13] Evans, A.E., Sawyer-Morse, M.K. and Betsinger, A. (2000) Fruit and Vegetable Consumption among Mexican-American College Students. Journal of the American Dietetic Association, 100, 1399-1402. http://dx.doi.org/10.1016/S0002-8223(00)00388-6

[14] Grace, T.W. (1997) Health Problems of College Students. Journal of American College Health, 45, 243-250. http://dx.doi.org/10.1080/07448481.1997.9936894

[15] Anding, J., Suminiski, R. and Boss, L. (2001) Dietary Intake, Body Mass Index, Exercise, and Alcohol: Are College Women Following the Dietary Guidelines? Journal of American College Health, 49, 167-171. http://dx.doi.org/10.1080/07448480109596299

[16] Shive, S.E. and Neyman Morris, M. (2006) Evaluation of the Energize Your Life! Social Marketing Campaign Pilot Study to Increase Fruit Intake among Community College Students. Journal of American College Health, 55, 33-39. http://dx.doi.org/10.3200/JACH.55.1.33-40

[17] Nelson, T.F., et al. (2007) Vigorous Physical Activity among College Students in the United States. Journal of Physical Activity \& Health, 4, 495-508.

[18] American College of Sports Medicine and American Heart Association (2007) Physical Activity and Public Health Guidelines. American College of Sports Medicine and American Heart Association, Indianapolis.

[19] (1998) Executive Summary of the Clinical Guidelines on the Identification, Evaluation, and Treatment of Overweight and Obesity in Adults. Archives of Internal Medicine, 158, 1855-1867. http://dx.doi.org/10.1001/archinte.158.17.1855

[20] Snyder, T., Dillow, S. and Hoffman, C. (2008) Digest of Education Statistics 2007 (NCES 2008-022). National Center for Education Statistics, Institute of Education Sciences, US Department of Education, Washington DC.

[21] Boyd, J.K. and Braun, K.L. (2007) Supports for and Barriers to Healthy Living for Native Hawaiian Young Adults Enrolled in Community Colleges. Preventing Chronic Disease, 4, 1-12.

[22] (1997) Youth Risk Behavior Surveillance, National College Health Risk Behavior Survey_United States, 1995. Morbidity and Mortality Weekly Report, 46, 1-56.

[23] Speakman, J.R., Walker, H., Walker, L. and Jackso, D.M. (2005) Associations between BMI, Social Strata and the Estimated Energy Content of Foods. International Journal of Obesity, 29, 1281-1288. http://dx.doi.org/10.1038/sj.ijo.0803018

[24] Merkin, S.S., Coresh, J., Roux, A.V.D., Taylor, H.A. and Powe, N.R. (2005) Area Socioeconomic Status and Progressive CKD: The Atherosclerosis Risk in Communities (ARIC) Study. American Journal of Kidney Disease, 46, 203-213. http://dx.doi.org/10.1053/j.ajkd.2005.04.033

[25] Loucks, E., Rehkopf, D.H., Thurston, R.C. and Kawachi, I. (2007) Socioeconomic Disparities in Metabolic Syndrome Differ by Gender: Evidence from NHANES III. Annals of Epidemiology, 17, $19-26$. http://dx.doi.org/10.1016/j.annepidem.2006.07.002

[26] Panagiotakos, D.B., et al. (2004) The Association between Educational Status and Risk Factors Related to Cardiovascular Disease in Healthy Individuals: The ATTICA Study. Annals of Epidemiology, 14, 188-194. http://dx.doi.org/10.1016/S1047-2797(03)00117-0

[27] Poddar, K.H., et al. (2009) Low-Fat Dairy Intake and Body Weight and Composition Changes in College Students. Journal of the American Dietetic Association, 109, 1433-1438. http://dx.doi.org/10.1016/j.jada.2009.05.005

[28] ACHA, American College Health Association (2009) American College Health Association-National College Health Assessment Spring 2008 Reference Group Data Report (Abridged). Journal of American College Health, 57, 477-488. http://dx.doi.org/10.3200/JACH.57.5.477-488 
[29] Kimm, S.Y., et al. (2002) Decline in Physical Activity in Black Girls and White Girls during Adolescence. The New England Journal of Medicine, 347, 709-715. http://dx.doi.org/10.1056/NEJMoa003277

[30] Wang, Y. and Beydoun, M.A. (2007) The Obesity Epidemic in the United States—Gender, Age, Socioeconomic, Racial/Ethnic, and Geographic Characteristics: A Systematic Review and Meta-Regression Analysis. Epidemiologic Reviews, 29, 6-28. http://dx.doi.org/10.1093/epirev/mxm007

[31] Berg, C.J., et al. (2011) The Development and Validation of a Scale Assessing Individual Schemas Used in Classifying a Smoker: Implications for Research and Practice. Nicotine \& Tobacco Research, 13, 1257-1265. http://dx.doi.org/10.1093/ntr/ntr144

[32] Centers for Disease Control and Prevention (2008) 2008 Physical Activity Guidelines for Americans. U.S. Department of Health and Human Services, Centers for Disease Control and Prevention, Atlanta.

[33] Cohen, S. and Lichtenstein, E. (1990) Perceived Stress, Quitting Smoking, and Smoking Relapse. Health Psychology, 9, 466-478. http://dx.doi.org/10.1037/0278-6133.9.4.466

[34] Diener, E., Emmons, R.A., Larsen, R.J. and Griffin, S. (1985) The Satisfaction With Life Scale. Journal of Personality Assessment, 49, 71-75. http://dx.doi.org/10.1207/s15327752jpa4901_13

[35] Gosling, S.D., Rentfrow, P.J. and Swann Jr., W.B. (2003) A Very Brief Measure of the Big-Five Personality Domains. Journal of Research in Personality, 37, 504-528. http://dx.doi.org/10.1016/S0092-6566(03)00046-1

[36] Hebl, M.R. and Heatherton, T.F. (1998) The Stigma of Obesity in Women: The Difference is Black and White. Personality and Social Psychology Bulletin, 24, 417-426. http://dx.doi.org/10.1177/0146167298244008

[37] de Bruijn, G.J., Kremers, S.P., van Mechelen, W. and Brug, J. (2005) Is Personality Related to Fruit and Vegetable Intake and Physical Activity in Adolescents? Health Education Research, 20, 635-644.

[38] Sheehan, K.B. (2001) E-mail Survey Response Rates: A Review. Journal of Computer-Mediated Communication, 6, 0.

[39] An, L.C., et al. (2007) Feasibility of Internet Health Screening to Recruit College Students to an Online Smoking Cessation Intervention. Nicotine \& Tobacco Research, 9, S11-S18.

\section{Abbreviations}

FV = fruit and vegetable; $\mathrm{PA}=$ physical activity; $\mathrm{BMI}=$ body mass index; $\mathrm{OR}=$ odds ratio; $\mathrm{CI}=95 \%$ confidence interval; PSS-4 = Perceived Stress Scale-4 item; TIPI = Ten Item Personality Inventory; CDC = Centers for Disease Control and Prevention 